\title{
OPIÓIDES E A COGNIÇÃO DE DOENTES COM DOR CRÔNICA: REVISÃO SISTEMÁTICA
}

\author{
Geana Paula Kurita, Cibele Andrucioli de Mattos Pimenta*, Moacyr Roberto Cuce Nobre
}

Trabalho realizado pelo curso de Pós-Graduação em Enfermagem na Saúde do Adulto da Escola de Enfermagem da Universidade de São Paulo, São Paulo, SP.

\author{
* Correspondência \\ Rua Dr. Enéas de Carvalho \\ Aguiar, n 419, Depto ENC \\ Cerqueira César, \\ São Paulo-SP, \\ CEP 05422-970 \\ Fax: (11) 30617544 \\ geanakurita@yahoo.com; \\ parpca@usp.br; \\ mrcnobre@usp.br
}

\begin{abstract}
RESUMO
OBjetivo. A crescente utilização de opióides em doentes com dor crônica de etiologias variadas e os possíveis efeitos desses fármacos em atividades diárias suscitam a importância de se avaliar tais efeitos. Com essa finalidade, desenvolveu-se este estudo de revisão sistemática que avaliou a influência de opióides nas funções cognitivas de doentes com dor crônica.

Métodos. Onze bases de dados foram analisadas utilizando-se os descritores opioids, opiates, narcotics, cognitive impairment, cognitive dysfunction, cognitive disorders e pain. Os critérios de inclusão foram: ensaios clínicos ou relatos de caso de dor crônica em tratamento com opióides, testes espećficos para avaliação cognitiva e publicação em inglês, espanhol ou português.

Resultados. Dezesseis pesquisas, publicadas entre 1980 e 2004, atenderam aos critérios: seis ensaios controlados, sendo dois deles randomizados, e dez estudos de menor força de evidência científica. Os ensaios de melhor qualidade, no contexto clínico do doente com dor crônica, principalmente na dor de origem não oncológica, mostraram que o uso de opióides não prejudica a função cognitiva.

Conclusã̃o. A ausência de prejuízo cognitivo com uso de opióides precisa ser confirmada em novos ensaios randomizados com número maior de pacientes com dor crônica.
\end{abstract}

UniteRMos: Dor. Câncer. Cognição. Opióides. Revisão sistemática.

\section{INTRODUÇÃo}

Os opióides são tradicionalmente recomendados para o controle da dor aguda e dor crônica oncológica, de moderada à intensa. Mais recentemente, em algumas situações, observa-se maior indicação para o controle da dor crônica não-oncológica.

O termo opióide é utilizado para denominar um grupo de drogas com propriedades semelhantes ao ópio. Denomina-se opiáceo os derivados naturais e alguns congêneres semi-sintéticos; e os sintéticos, de opióides. No entanto, há a tendência de se generalizar o termo opióide para todas as substâncias que atuam por meio da interação com receptores opióides. A denominação "narcóticos" deve ser preterida para dissociar-se da idéia de sono e torpor, pois a principal ação dos opióides é a analgesia!.

A ação analgésica dá-se em sítios espinhais e supra-espinhais. Os opióides inibem a transmissão ascendente dos estímulos nociceptivos, provenientes do corno dorsal da medula espinhal, e ativam as vias de modulação da dor, que descendem do mesencéfalo pelo bulbo ventromedial rostral e chegam ao corno dorsal da medula espinhal!.

Os efeitos dos opióides no Sistema Nervoso Central são diversos e não completamente conhecidos. Acredita-se que possam interferir na aquisição, processamento, armazenamento e recuperação da informação ${ }^{2}$ e acarretar alterações psicomotoras, do humor (euforia e disforia), da concentração, da memória e do tempo de reaçãa, 3,4 . Alterações cognitivas podem comprometer o funcionamento dos doentes em suas atividades físicas, laborativas, sociais e de lazer. Estudos com voluntários sadios com pouca ou nenhuma exposição a drogas psicoativas, demonstraram grande tendência à disfunção cognitiva após a administração pontual de opióides ${ }^{5}$.

O consumo de opióides cresceu entre $26 \%$ e $1423 \%$ em países como a Dinamarca, Suíça, Austrália, Estados Unidos, Holanda, Reino Unido, Suécia, Nova Zelândia, Canadá e Israel. Uma análise de 1.854 prescrições dinamarquesas feitas por clínicos gerais revelou que menos de $10 \%$ foram aviadas para o controle da dor oncológica 6 .

A prescrição de opióides para doentes com história de câncer avançado é unânime na área médica e seus possíveis efeitos deletérios sobre a função cognitiva parecem não representar preocupação relevante. A utilização de opióides em doentes com doença oncológica não avançada, com maior expectativa de vida e o aumento na indicação de opióides para o controle da dor crônica nãooncológica despertam interesse em se refinar o conhecimento sobre os efeitos desses medicamentos na função cognitiva de doentes com dor crônica.

\section{Métodos}

Trata-se de uma revisão sistemática em que se objetivou analisar os estudos sobre os efeitos dos opióides na função cognitiva em pessoas com dor crônica. O levantamento bibliográfico foi realizado em março de 2004 e revisado no fim do primeiro semestre de 2005 e abrangeu toda a base Psiperiodicos, PubMed, Medline, Lilacs, Cinahl, Cochrane, Web of Science, Psyinfo, Dissertation Abstracts, Drug Information e Evidence Based Medicine. A pergunta para a realização da revisão sistemática foi estruturada com três dos quatro 
Tabela I - Distribuição dos 16 estudos sobre doentes com dor crônica, não oncológica e oncológica, segundo os efeitos dos opióides sobre a função cognitiva,

\begin{tabular}{|c|c|c|c|}
\hline Autor/ano & Desenho* & $\begin{array}{c}\text { Nível de } \\
\text { evidência/grau recomendação* }\end{array}$ & Momento de avaliação \\
\hline \multicolumn{4}{|l|}{ Dor não-oncológica } \\
\hline Raja et al $(2002)^{8}$ & ECR & $\mathrm{Ib} / \mathrm{A}$ & Antes e depois de 8 semanas \\
\hline Moulin et al $(1996)^{9}$ & ECR & $\mathrm{Ib} / \mathrm{A}$ & Antes, e depois (4x) \\
\hline Haythornthwaite et al (1998) ${ }^{10}$ & ECC & $2 C / B$ & Antes e depois de dose estabilizada \\
\hline Hendler et al (1980)" & ECC & $2 c / B$ & Após opióide \\
\hline Tassain et al $(2003)^{12}$ & ECNC & $2 C / B$ & Antes, 3, 6, 12 meses após \\
\hline Meneffee et al $(2004)^{13}$ & ECNC & $2 C / B$ & Antes e depois (após 4 semanas de estabilização da dose) \\
\hline Jamison et al $(2003)^{14}$ & ECNC & $2 C / B$ & Antes, após 90 e 180 dias \\
\hline Lorenz et al $(1997)^{15}$ & $\mathrm{RC}$ & $3 b / B$ & Antes e após alívio da dor \\
\hline Galski et al $(2000)^{16}$ & $\mathrm{RC}$ & $3 b / B$ & Após opióide \\
\hline Sabatowski et al $(2003)^{17}$ & EVGC & $2 C / B$ & Após 12 dias de dose estável \\
\hline Sjøgren et al $(2000)^{18}$ & EVGC & $2 C / B$ & Após 14 dias de dose estável \\
\hline \multicolumn{4}{|l|}{ Dor oncológica } \\
\hline Sjøgren et al $(2000)^{19}$ & ECC & $2 C / B$ & + 2 semanas em uso de opióides (40mg - 120mg) \\
\hline
\end{tabular}

\begin{tabular}{|c|c|c|c|}
\hline Clemons et al $(1996)^{20}$ & ECC & $2 c / B$ & $\begin{array}{l}\text { I,5h após ingestão oral ou th após morfina de liberação prolongada } \\
\text { (após dose estável) }\end{array}$ \\
\hline Pita $(1998)^{21}$ & ECNC & $2 C / B$ & Antes, após 1/7/90 dias \\
\hline Vainio et al $(1995)^{22}$ & EOSDC & $2 C / B$ & Mínimo de 2 semanas com dose estável morfina vo \\
\hline Sjøgren et al $(1989)^{23}$ & EVGC & $2 c / B$ & Mínimo de 2 semanas com dose estável morfina vo \\
\hline
\end{tabular}

$E C R=$ ensaio clinico randomizado $\quad E C C=$ ensaio clínico controlado $E C N C=$ ensaio clínico não controlado $\quad R C=$ relato de casos $\quad E V G C=$ ensaio com voluntários no grupo de comparação EOSDC=ensaio com paciente oncológico sem dor no grupo de comparação * Classificação adotada pelo Oxford Centre for Evidence-based Medicine

elementos do PICO conceituado pela Medicina Baseada em Evidência. Paciente: com dor crônica. Intervenção: uso de analgésico opióide. Outcome/desfecho avaliado: presença de alteração cognitiva. Não foi incluído o elemento controle para ampliar o número de artigos recuperados. Essa pergunta de pesquisa gerou a seguinte estratégia para busca em bases de dados informatizadas: pain AND (opioids or opiates or narcotics)AND (cognitive impairment OR cognitive dysfunction OR cognitive disorders OR memory OR attention $O R$ concentration OR reaction time)
Foram identificados 150 estudos. Pela análise do resumo foram selecionados os que apresentavam o delineamento do tipo ensaio clínico ou estudos observacionais; que atendiam aos critérios de inclusão de ter sido realizado em doentes com dor crônica, ter utilizado teste padronizado para a avaliação das funções cognitivas; e finalmente, estar escrito em inglês, espanhol ou português. Resultaram 16 artigos escritos em língua inglesa, todos publicados entre 1980 e 2004, que estão descritos em duas seções. A primeira contém os estudos sobre os efeitos dos opióides na função cognitiva em doentes com dor crônica de origem não oncológica, 
com o desenho metodológico, momento em que ocorreu a avaliação e existência de grupo controle ou de comparação. São Paulo, 2005

Grupo controle ou de comparação Função cognitiva

76 doentes com dor avaliados em uso de opióide e placebo

46 doentes com dor avaliados em uso de opióide e placebo psicoativo (benztropina)

19 doentes c/opióide

10 doentes s/opióide

61 doentes $\mathrm{c} /$ benzodiazepínico +opióide, 21 doentes c/opióides, 18 doentes $\mathrm{Cl}$ benzodiazepínicos, 6 doentes sem medicamento

18 doentes c/opióide

10 doentes interromperam tratamento por efeito colateral

$-$

$16 \mathrm{c} / \mathrm{dor}$

327 doentes neurológicos

30 doentes $\mathrm{c} /$ dor

90 sadios

40 doentes $\mathrm{c} / \mathrm{dor}$

40 sadios

130 doentes

40 s/dor s/opióide, 19 s/dor s/opióide,

19 c/dor s/opióide, 31 c/dor c/opióide,

21 s/dor c/opióide

16 sadios

6 doentes com câncer

7 doentes $\mathrm{c} /$ morfina

$-$

24 c/dor c/opióide

25 s/dor s/opióide

14 doentes

20 Sadios
Sem alteração

Sem alteração

Melhora da velocidade psicomotora e da atenção

Sem alteração

Melhora do processo da informação, do humor, da velocidade e coordenação motora

Melhora da memória, da atenção e da velocidade motora

Melhora da concentração, da velocidade motora e mental

Melhora do estado de alerta e tempo de reação

Sem alteração

Sem alteração

Piora da vigilância, atenção, memória e velocidade psicomotora

Sem alteração

Sem alteração

Piora da memória

Piora do equilibrio

Melhora da movimentação psicomotora (autores desconsideraram essas alterações)

Piora no tempo de reação e a segunda apresenta as pesquisas realizadas com doentes com dor de origem neoplásica. Em adição, os estudos foram classificados pelo desenho da pesquisa, de acordo com o Oxford Centre for Evidence-based Medicine ${ }^{7}$ em nível de evidência de I a 5 ( Ia, Ib, Ic, 2a, 2b, 2c, 3a, 3b, $4,5)$ e grau de recomendação de $A$ a $D$, os quais refletem a consistência da evidência em ordem decrescente de poder .

Não foi possível a realização de metanálise, visto os diferentes desenhos de pesquisa, diversidade de testes para avaliação cognitiva e de amostras estudadas.

\section{Resultados}

\section{Efeitos dos opióides na função cognitiva de doentes com dor crônica não neoplásica}

Foram analisados I I trabalhos em doentes com dor não oncológica (Tabela I). Desses, dois foram ensaios randomizados, do tipo crossover e duplo-cego ${ }^{8,9}$, dois foram ensaios clínicos controlados ${ }^{10,11}$, três foram ensaios clínicos não-controlados ${ }^{12-14}$, dois foram relatos de caso $^{15,16}$, dois estudos foram classificados como ensaios que usaram voluntários saudáveis para a formação do grupo de comparação ${ }^{17,18}$. 


\begin{tabular}{|c|c|c|c|c|}
\hline \multicolumn{5}{|c|}{ Tabela 2 - Descrição dos estudos realizados com doentes com dor crônica de origem não oncológica } \\
\hline $\begin{array}{l}\text { Autor/ano } \\
\text { dor não-oncológica }\end{array}$ & $\mathbf{N}$ & $\begin{array}{c}\text { Idade } \\
\text { Média (variação) }\end{array}$ & $\begin{array}{l}\text { Sexo } \\
M / F\end{array}$ & Fármaco \\
\hline Raja et al $(2002)^{8}$ & 76 doentes & $\begin{array}{l}71,0 \\
\text { (nd) }\end{array}$ & $34 / 42$ & $\begin{array}{l}\text { Morfina } \\
\text { Metadona } \\
\text { Nortriptilina } \\
\text { Desipramina }\end{array}$ \\
\hline Moulin et al $(1996)^{9}$ & 46 doentes & $\begin{array}{c}40,4 \\
(26-67)\end{array}$ & $19 / 27$ & Morfina de liberação prolongada \\
\hline Haythornthwaite et al $(1998)^{10}$ & $\begin{array}{l}19 \text { doentes c/opióide } \\
10 \text { s/opióide }\end{array}$ & $\begin{array}{l}50,8 \\
52,2 \\
(\mathrm{nd})\end{array}$ & $\begin{array}{l}8 / 11 \\
2 / 8\end{array}$ & Opióides de liberação prolongada \\
\hline Hendler et al $(1980)^{\prime \prime}$ & 106 doentes & $\begin{array}{c}43,4 \\
(22-69)\end{array}$ & nd & $\begin{array}{c}\text { Opióides } \\
\text { Benzodiazepínico }\end{array}$ \\
\hline Tassain et al $(2003)^{12}$ & $\begin{array}{l}18 \text { doentes c/opióide } \\
10 \text { doentes s/opióide }\end{array}$ & $\begin{array}{c}46 \\
(18-65) \\
51,4 \\
(27-65)\end{array}$ & $\begin{array}{l}10 / 8 \\
1 / 9\end{array}$ & Morfina de liberação prolongada \\
\hline Meneffee et al $(2004)^{13}$ & 23 doentes & $\begin{array}{c}47,0 \\
(18-67)\end{array}$ & $6 / 17$ & $\begin{array}{l}\text { Fentanil } \\
\text { Oxicodona }\end{array}$ \\
\hline Jamison et al $(2003)^{14}$ & I44 doentes & $\begin{array}{l}46,3 \\
(n d)\end{array}$ & $99 / 45$ & $\begin{array}{l}\text { Oxicodona } \\
\text { Acetaminofeno } \\
\text { Fentanil }\end{array}$ \\
\hline Lorenz et al $(1997)^{15}$ & 6 doentes & $\begin{array}{c}50,7 \\
(38-61)\end{array}$ & nd & Morfina de liberação prolongada \\
\hline Galski et al $(2000)^{16}$ & $\begin{array}{l}16 \mathrm{c} / \text { dor } \\
327 \text { doentes neurológicos }\end{array}$ & $\begin{array}{l}48,9 \\
46 \\
(n d)\end{array}$ & nd & $\begin{array}{c}\text { Opióides de ação prolongada e curta } \\
\text { Morfina ou fentanil }\end{array}$ \\
\hline Sabatowski et al $(2003)^{17}$ & $\begin{array}{l}30 \text { doentes } \\
90 \text { sadios }\end{array}$ & $\begin{array}{c}50,0 \\
(18-65) \\
50,0 \\
(18-80)\end{array}$ & $\begin{array}{l}18 / 12 \\
57 / 33\end{array}$ & Fentanil \\
\hline Sjøgren et al $(2000)^{18}$ & $\begin{array}{l}40 \text { doentes } \\
40 \text { sadios }\end{array}$ & $\begin{array}{c}60,0 \\
(46-74) \\
59,0 \\
(49-78)\end{array}$ & $\begin{array}{l}16 / 24 \\
11 / 29\end{array}$ & $\begin{array}{c}\text { Morfina de liberação prolongada } \\
\text { Metadona } \\
\text { Buprenorfina } \\
\text { Tramadol }\end{array}$ \\
\hline
\end{tabular}

Os desenhos dos estudos de melhor qualidade ${ }^{8,9}$, controlados e randomizados, mostraram que pacientes com dor crônica nãooncológica em uso de opióide não apresentam alteração da função cognitiva. A maioria dos estudos de menor qualidade, nãorandomizados, também não referiu alteração da função cognitiva ${ }^{10-17}$, sendo que alguns mostraram melhora de algumas funções, como a atenção/concentração e velocidade psicomotora ${ }^{10,12-15}$. Somente um estudo que utilizou voluntários no grupo de comparação concluiu pela piora na atenção e memória dos pacientes tratados com opióides $^{18}$ (Tabela I).

As avaliações ocorreram após o início do tratamento com opióides em quatro estudos ${ }^{11,16-18}$ e em sete, antes e após o início do opóide ${ }^{8-}$ 10,12-15. Nos estudos em que foi utilizado outro grupo para comparação, um foi de doentes neurológicos sem dor ${ }^{16}$, três com doentes em tratamento com drogas não opióides ${ }^{8,9.11}$, e dois utilizaram voluntários $\operatorname{sadios}^{17,18}$ (Tabela I). 
que receberam opióides e a influência nas funções cognitivas ( $\mathrm{n}=$ I I estudos). São Paulo, 2005.

\section{média ou mínima e/ou máxima}

$91 \mathrm{mg} / \mathrm{dia}$

$15 \mathrm{mg} / \mathrm{dia}$

$98 \mathrm{mg} / \mathrm{dia}$

$63 \mathrm{mg} / \mathrm{dia}$

$120 \mathrm{mg} / \mathrm{dia}$
máx

22,8mg a | II, Img/dia

oral

oral

$40 \mathrm{mg}$ a $140 \mathrm{mg} / \mathrm{dia}$

oral

$$
\begin{aligned}
& 125 \mu g / h o r a \\
& 15 \mathrm{mg} / \mathrm{dia} \\
& \text { máx }
\end{aligned}
$$

$32,7 \mathrm{mg} / \mathrm{dia}$ a $38,2 \mathrm{mg} / \mathrm{dia}$

$42,6 \mu g / d i a$ a $43 \mu g / d i a$

150mg/dia máx

$$
30 \mathrm{mg}
$$

mín

50ugh

$60 \mathrm{mg} / \mathrm{dia}$ transdérmica

oral

transdérmica

oral

oral transdérmica

transdérmica

oral
Instrumentos para avaliação cognitiva

Wechsler Adult Intelligence Scale-Revised, Hopkins Verbal Learning

Test, Beck Depression Inventory, Profile of Mood States

Sympton Check List-90, Profile of Mood States, High Sensitivity Cognitive Screen

Beck Depression Inventory, Grooved Pegboard, Hopkins Verbal Learning

Test, Trail Making Test, Digit Symbol, Digit Span subscale - Wechsler Adult Intelligence Test

Wechsler Adult Intelligence Scale, Memory Quotient, Bender-Gestalt Test

Reminding Test, Stroop Task, Trail Making Test, Digit Span Test, Digit Symbol subtest- Wechsler Adult Intelligence Scale, free and cued selective remind test (memory, verbal fluency and reaction time)

Trail Making Test, Rey Complex Figure Test and Recognition Trial, Weschler Memory Scale-III Spatial Span Test, d2 Test of Attention, Conners' Continuous Performance Test II

Beck Depression Inventory, Digit Symbol Substitution Test, Trail Making Test Form B

Oddball Paradigm, Vonzerssen Scales

Digit Symbol e Block Design subtest subtests - Wechsler Adult Intelligence Scale-Revised, Trail Making Test-A, Double Letter Cancellation Test, Visual Form Recognition Test, Rey-Osterreith Complex Figure Test, Ravens Progressive Matrices, Porteus Maze Test

Attention test, Test for reaction time under pressure, determination test, test for visual orientation, tachistoscopic perception, test for motor co-ordination, vigiance test, Passed test

Continuous reaction time, finger tapping test, Paced Auditory Serial Addition

Task, Hospital Anxiety and Depression Scale
Além da variabilidade dos estudos quanto ao desenho, observouse grande variabilidade quanto ao número de sujeitos estudados, tipo e dose de opióides, e testes utilizados para avaliação cognitiva. A via de administração foi a oral ou a transdérmica (Tabela 2).

A evidência de maior nível observada foi gerada por dois estudos controlados e randomizados ${ }^{8,9}$, nos quais os doentes com opióide não apresentaram diferenças na função cognitiva. Entretanto, um dos estudos utilizou um placebo psicoativo9 (Tabela I).

\section{Ação dos opióides na função cognitiva de doentes com dor crônica oncológica}

Foram analisados cinco estudos que avaliaram doentes com dor oncológica (Tabela I). Desses, dois ensaios clínicos controlados ${ }^{19,20}$, um ensaio não-controlado ${ }^{21}$, um ensaio controlado com paciente oncológico sem dor ${ }^{22}$ e um ensaio que usou como comparação individuos voluntários saudáveis ${ }^{23}$ (Tabela I). 
Tabela 3 - Descrição resumida dos estudos realizados com doentes com dor crônica de origemoncológica

\begin{tabular}{|c|c|c|c|c|}
\hline $\begin{array}{l}\text { Autor/ ano } \\
\text { dor oncológica }\end{array}$ & $\mathbf{N}$ & $\begin{array}{c}\text { Idade } \\
\text { Média (variação) }\end{array}$ & $\begin{array}{l}\text { Sexo } \\
M / F\end{array}$ & Fármaco \\
\hline Sjøgren et al $(2000)^{19}$ & $\begin{array}{l}G 1=40 \text { s/dor s/opióide } \\
G 2=19 \text { s/dor s/opióide } \\
G 3=19 \mathrm{c} / \text { dor s/opióide } \\
G 4 a=31 \mathrm{c} / \text { dor c/opióide } \\
\text { G4b=21 s/dor c/opióide }\end{array}$ & $\begin{array}{l}62,5(49-73) \\
63,0(40-75) \\
58,0(46-76) \\
59,0(47-74) \\
60,0(46-73)\end{array}$ & $\begin{array}{l}19 / 21 \\
15 / 4 \\
13 / 6 \\
21 / 10 \\
12 / 9\end{array}$ & $\begin{array}{c}\text { nd } \\
\text { doses equivalentes à morfina }\end{array}$ \\
\hline Clemons et al $(1996)^{20}$ & $\begin{array}{c}6 \text { doentes s/ opióide } \\
7 \text { doentes c/ opióide } \\
16 \text { sadios }\end{array}$ & $\begin{array}{l}61,0(41-76) \\
65,4(49-83) \\
62,8(44-90)\end{array}$ & $\begin{array}{l}2 / 5 \\
8 / 8 \\
3 / 3\end{array}$ & Morfina \\
\hline Pita $(1998)^{21}$ & 20 doentes & $62(32-78)$ & $11 / 9$ & Morfina \\
\hline Sjøgren et al $(1989)^{23}$ & $\begin{array}{l}14 \text { doentes } \\
20 \text { sadios }\end{array}$ & $\begin{array}{l}60,0(44-71) \\
56,0(43-64)\end{array}$ & nd & Morfina \\
\hline
\end{tabular}

Quanto à avaliação, apenas um estudo avaliou os doentes antes do início do tratamento com opióide ${ }^{21}$ (Tabela I).

Os ensaios clínicos controlados mostraram que o uso do opióide não alterou a função cognitiva ${ }^{19,20}$. Os estudos sem grupo de comparação ${ }^{21}$, ou no qual a análise comparativa foi realizada com sadios $^{23}$ ou doentes sem dor e sem opióide ${ }^{22}$, mostraram piora da memória, equilibrio, e no tempo de reação (Tabela I).

Observou-se que os poucos estudos encontrados diferiram quanto ao desenho, tiveram grande variabilidade quanto ao número de sujeitos e testes utilizados para avaliação cognitiva. A morfina foi utilizada por via oral em quatro estudos ${ }^{19,20,22,23}$ (Tabela 3).

Entre os estudos de menor grau de recomendação aquele que teve maior amostra e grupos de comparação diversificados mostrou que os doentes com opióide não apresentaram diferenças na função cognitiva $^{19}$ (Tabela I).

\section{Discussão}

Após extenso levantamento bibliográfico, encontrou-se pequeno número de estudos sobre o tema, com diferentes desenhos. Os estudos mais bem desenhados não indicam prejuízo da função mental (grau de recomendação A e B). Nos estudos de melhor qualidade (melhor desenho, maior amostra, grupo controle adequado), não se observou alteração da função cognitiva. Considerando-se que a dor pode ser causa de prejuízo às funções mentais, é possível que a analgesia, decorrente do uso do opióide, possa ter auxiliado na manutenção das funções cognitivas.

Nos II estudos com doentes com dor crônica não-oncológica, houve equilíbrio entre a ausência de efeitos em cinco e efeitos benéficos sobre a cognição em outros cinco. Nos cinco estudos com doentes com dor crônica de origem neoplásica, não houve alteração cognitiva nos estudos de melhor qualidade. A identificação da causa da disfunção cognitiva é difícil pela concomitância de fatores relacionados à doença e ao tratamento como o acometimento de múltiplos sistemas e o uso de outros medicamentos psicoativos.

Analisando-se o conjunto dos estudos, observa-se que os dados são insuficientes para conclusões mais definitivas, mas apontam para não prejuízo ou melhora da função cognitiva em doentes com dor crônica em tratamento com opióides. Embora os estudos sejam classificáveis pelo nível de evidência Oxford, há que se atentar para as limitações metodológicas.

A maioria dos estudos analisados foram realizados com amostras pequenas e de conveniência, o que pode ter causado algum viés na interpretação dos dados. $\bigcirc$ grupo controle em alguns estudos não foi o mais adequado, doentes com dor podem ter redução da habilidade mental devido a própria dor, enquanto pessoas sadias podem ter melhor desempenho cognitivo pela ausência de doença. Em muitos estudos a avaliação cognitiva ocorreu em doentes que já faziam uso de opióides, o que impede o conhecimento do estado cognitivo do doente antes do tratamento. A coleta de dados longitudinal com várias avaliações ocorreu em poucos estudos, o que suscita dúvidas sobre o efeitos dos opióides a longo prazo. Ainda, houve variabilidade no uso dos opióides, o que levanta questionamentos sobre diferentes efeitos que cada um desses fármacos pode causar.

Há referência na literatura que a dor por si só pode ser causa de alteração cognitiva ${ }^{19}$. $\bigcirc$ sofrimento emocional e o estresse gerados por ela, independente da intensidade, podem resultar em prejuízos. Além disso, respostas fisiológicas ao estresse talvez tenham ação desregulatória no eixo hipotálamo-pituitário-adrenocortical, o que 
que receberam opióides e a influência nas funções cognitivas ( $n=5$ estudos). São Paulo, 2005.

\begin{tabular}{|c|c|c|}
\hline $\begin{array}{c}\text { Dose } \\
\text { média ou mínima e máxima }\end{array}$ & Via & Instrumentos para avaliação cognitiva \\
\hline $\begin{array}{l}120 \mathrm{mg} / \mathrm{dia} \\
40 \mathrm{mg} / \mathrm{dia} \\
\text { (medianas) }\end{array}$ & oral & $\begin{array}{l}\text { Sinais auditivos usados na mensuraçãa do tempo de reação, } \\
\text { Finger Tapping Test, Paced Auditory Serial Addition Task }\end{array}$ \\
\hline
\end{tabular}

\begin{tabular}{|c|c|c|}
\hline $50 \mathrm{mg}$ a 200mg/dia & oral & $\begin{array}{l}\text { New Adult Reading Test, Logical Memory Test, Grammatical Reasoning Test, Reaction Time, } \\
\text { Stroop Colour-word test, Visual Analogue Scales, University of Wales } \\
\text { Institute of Science \& Technology Mood Adjective Checklist }\end{array}$ \\
\hline $0,25 \mathrm{mg}$ a $4,0 \mathrm{mg} / \mathrm{dia}$ & intraventricular & $\begin{array}{c}\text { NAl-figuren Test, Wechsler Memory Scale, Beck's Depression Tests, } \\
\text { teste formulado pelo pesquisador }\end{array}$ \\
\hline 209mg/dia & oral & $\begin{array}{l}\text { Finger Tapping, Reaction Time, Middlesex } \\
\text { method, Body Sway, Wartegg Personality Test }\end{array}$ \\
\hline $\begin{array}{l}130 \mathrm{mg} \text { a } 400 \mathrm{mg} / \mathrm{dia} \\
32 \mathrm{mg} \text { a } 240 \mathrm{mg} / \mathrm{dia}\end{array}$ & $\begin{array}{l}\text { oral } \\
\text { epidural }\end{array}$ & Sinais auditivos usados na mensuraçãão do tempo de reação \\
\hline
\end{tabular}

contribui para a disfunção cognitiva ${ }^{24}$. A intensidade da dor parece interferir na cogniçãão $25-27$ e seu efeito parece mais evidente nos primeiros dias de uso do opióide ${ }^{25}$. O alívio da dor é considerado importante para a melhora no desempenho cognitivo, embora não haja unanimidade sobre o tamanho desse efeito.

Para alguns autores o uso de opióides por doentes com dor crônica parece ser seguro do ponto de vista cognitivo ${ }^{5}$ e não impossibilitar a condução de veículos motorizados ${ }^{28}$. No entanto, há preocupações sobre a generalização da informação que opióide não interfere na função cognitiva de doentes com dor crônica, visto que um único caso de disfunção cognitiva pode ocasionar acidentes sérios $^{25}$.

O prejuízo dos opióides na função cognitiva é ainda controverso. Alguns pesquisadores revisaram estudos sobre os efeitos dos opióides na função cognitiva de doentes com dor crônica e observaram dificuldades em mensurar esses efeitos, pois são fáceis de serem decorrentes de complicações da doença oncológica ${ }^{29}$, do uso concomitante de outros psicofármacos ${ }^{25}$, da idade, da escolaridade e da própria dor, entre outros. Ainda, esses fatores interferem nos aspectos metodológicos e, conseqüentemente, limitam os resultados ${ }^{30}$.

Para se analisar os efeitos dos opióides sobre a cognição, há necessidade de se comparar grupo com dor recebendo opióides, grupo com dor recebendo analgésicos não-opióides e grupo sem dor. Há que se ter avaliações cognitivas antes do início do uso dos opióides, controlar a evolução da dor e controlar as outras situações que possam levar a piora na função cognitiva, como o agravamento da doença, ocorrência de metástase cerebral, piora na condição física como anemia e desidratação, entre outros. É preciso separar os efeitos do opióide dos efeitos da dor sobre a cognição, entre outras situações. Estudo que atenda a essas características não foi encontrado na literatura analisada, é de realização difícil, mas fundamental.

A maior parte dos estudos não possuía desenho que permitisse essa diferenciaçãa. As amostras foram pequenas; em muitos não houve avaliação da função cognitiva antes do início do tratamento com o opióide para se estabelecer o estado mental basal do doente; a variedade de instrumentos utilizados foi muito grande e foram utilizados diversos fármacos em doses e vias variadas. Tais aspectos impossibilitam comparações e conclusões mais definitivas.

\section{Conclusão}

O conhecimento sobre alteração cognitiva em doentes com dor crônica recebendo opióides está em construção e os poucos estudos existentes com maior nível de evidência indicam, na maior parte dos casos, ausência de alteração ou melhora cognitiva, provavelmente mediada pelo alívio da dor. No entanto, as limitações dos estudos impõem cautela nas conclusões. Considerando-se que os dados empíricos são insuficientes, que o uso de opióides para o controle da dor é crescente, principalmente em doentes que continuam trabalhando e realizando atividades do dia-a-dia, investigações com método mais aprimorado são necessárias.

\section{Nota}

O presente trabalho foi realizado com o apoio da CAPES, entidade do Governo Brasileiro voltada para a formação de recursos humanos.

\section{Conflito de interesse: não há}




\section{SUMMARY \\ OpIOIDS AND COGNITION BY PATIENTS WITH CHRONIC PAIN: A SYSTEMATIC REVIEW}

OBIECTIVE. The increasing use of opioids by patients suffering from chronic pain caused by different etiologies, and the possible effects of those substances on everyday activities, require careful evaluation of their effects. For this purpose, a systematic review was developed to assess the influence of opioids on the cognitive function in patients with chronic pain.

METHODS. Eleven databases were analyzed using the following descriptors: opioids, opiates, narcotics, cognitive impairment, cognitive dysfunction, cognitive disorders and pain. The inclusion criteria were: clinical trials or case reports which included patients with chronic pain in treatment with opioids, cognitive assessment by specific tests and publication in English, Spanish or Portuguese.

RESULTS. Sixteen surveys published between 1980 and 2004 met the criteria: six controlled trials, two randomized trials, and 10 studies of a lower scientific evidence level. All better quality controlled trials did not show cognitive impairment of patients under opioid therapy.

CONCLUSION. These results must be confirmed by additional randomized trials including a greater number of patients with chronic pain. [Rev Assoc Med Bras 2008; 54(6): 529-36]

KEY words: Pain. Cancer. Cognition. Opioids. Systematic review.

\section{REFERÊNCIAS}

I. Gutstein HB, Akil H. Analgésicos opióides. In: Gilman AG, Hardman JG, Limbird LE, editores. Goodman \& Gilman: as bases farmacológicas da terapêutica. $10^{\text {a }}$ ed. Rio de Janeiro: McGraw-Hill; 2003. p.427-64.

2. Lawlor PG. The panorama of opiod-related cognitive dysfunction in patients with cancer. Cancer. 2002;94:1836-53.

3. Daeninck PJ, Bruera E. Opiod use in cancer pain: is a more liberal approach enhancing toxicity? Acta Anaesthesiol Scand. 1999;43:924-38.

4. Bruera E, Pereira J. Recent developments in palliative cancer care. Acta Oncol. 1998:37:749-57.

5. Zacny JP. A review of the effects of opioids on psychomotor and cognitive functioning in humans. Exp Clin Psychopharmacol. 1995;3:432-66.

6. Clausen TG. International opioid consumption. Acta Anaesthesiol Scand. 1997:4 |: |62-5

7. Phillips B, Ball C, Sackett DL, Badenoch D, Straus S, Haynes B, Dawes M. Oxford Centre for Evidence-based Medicine Levels of Evidence, $200 \mathrm{I}$. [cited 2008 feb l0]. Available from: http://www.cebm.net/ index.aspx?o $=1025$.

8. Raja SN, Haythornthwaite JA, Pappagallo M, Clark M, Travison G, Sabeens S. Opioids versus antidepressants in postherpetic neuralgia: a randomized, placebo-controlled trial. Neurology. 2002;59: I | I 5-2 I.

9. Moulin DE, lezzi A, Amireh R, Sharpe WK, Boyd D, Merskey H. Randomised trial of oral morphine for chronic non-cancer pain. Lancet. 1996;347: I 43-7.

10. Haythorthwaite JA, Menefee LA, Quatrano-Piacentini AL. Outcome of chronic opiod therapy for non-cancer pain. J Pain Symptom Manage. 1998; 15:185-94.

II. Hendler N, Cimini C, MA T, Long D. A comparison of cognitive impairment due to benzodiazepines and to narcotics. Am J Psychiatry. 1980; 137:828-30.
12. Tassain $\mathrm{V}$, Attal N, Fletcher D, Brasseur L, Dégueux P, Chauvin M, et al. Long term effects of oral sustained release morphine on neuropsychological performance in patients with chronic non-cancer pain. Pain. 2003; 104:389-400.

13. Menefee LA, Frank ED, Crerand C, Jalali S, Park J, Sanschagrin K, et al. The effects of transdermal fentanyl on driving, cognitive performance, and balance in patients with chronic nonmalignant pain conditions. Pain Med. 2004;5:42-9

14. Jamison RN, Schein JR, Vallow S, Ascher S, Vorsonger GJ, Katz NP. Neuropsychological effects of long-term opiod use in chronic pain patients. J Pain Symptom Manage. 2003;26:913-21.

15. Lorenz J, Beck H, Bromm B. Cognitive performance, mood and experimental pain before and during morphine-induced analgesia in patients with chronic non-malignant pain. Pain. 1997;73:369-75.

16. Galski T, Williams B, Ehle HT. Effects of opioids on driving ability. J Pain Symptom Manage. 2000; 19:200-8.

17. Sabatowski R, Schwalen S, Rettig K, Herberg KW, Kasper SM, Radbruch L. Driving ability under long-term treatment with transdermal fentanyl. J Pain Symptom Manage. 2003;25:38-47.

18. Sjøgren P, Thomsen AB, Olsen AK. Impaired neuropsychological performance in chronic nonmalignant pain patients receiving long-term oral opioid therapy. J Pain Symptom Manage. 2000; 19: I00-8.

19. Sjøgren P, Olsen AK, Thomsen AB, Dalberg J. Neuropsychological performance in cancer patients: the role of oral opioids, pain and performance status. Pain. 2000;86:237-45.

20. Clemons M, Regnard C, Appleton T. Alertness, cognition and morphine in patients with advanced cancer. Cancer Treat Rev. 1996;22:45I-68

21. Pita G. Disturbances in recent memory and behavioural changes caused by the treatment with intraventricular morphine administration (IVM) in severe cancer pain. Hum Psychopharmacol Clin Exp. 1998; | 3:3 | 5-23.

22. Vainio A, Ollila J, Matikainen E, Rosenberg P, Kalso E. Driving ability in cancer patients receiving long-term morphine analgesia. Lancet. 1995;346:667-70.

23. Sjøgren $P$, Banning A. Pain, sedation and reaction time during long-term treatment of cancer patients with oral and epidural opioids. Pain. 1989:39:5-11.

24. Hart RP, Wade JB, Martelli MF. Cognitive impairment in patients with chronic pain: the significance of stress. Curr Pain Headache Rep. 2003;7:116-26.

25. Chapman SL, Byas-Smith MG, Reed BA. Effects of intermediate and longterm use of opioids on cognition in patients with chronic pain. Clin J Pain. 2002; I 8:S83-590.

26. Kurita GP. Alteração cognitiva e o tratamento da dor oncológica. [tese] São Paulo: Escola de Enfermagem. Universidade de São Paulo; 2006.

27. Kurita GP, Pimenta CAM. Cognitive impairment in cancer pain patients receiving opioids: a pilot study. Cancer Nurs. 2008;30:49-57 .

28. Kress HG, Kraft B. Opioid medication and driving ability. Eur J Pain. 2005;9:141-4.

29. O'Neill WM. The cognitive and psychomotor effects of opioid drugs in cancer pain management. Cancer Surv. 1994;21:67-84.

30. Ersek M, Cherrier MM, Overman SS, Irving GA. The cognitive effects of opioids. Pain Manage Nurs. 2004;5:75-93.

Artigo recebido: 27/02/07

Aceito para publicação: 24/03/08 